\title{
Neurological deterioration or symptom progression in Wilson's disease after starting zinc sulphate treatment - a
} case report

\author{
Karolina Dzieżyc ${ }^{1 *}$, Tomasz Litwin ${ }^{1}$ and Anna Członkowska ${ }^{1,2}$ \\ ${ }^{1}$ Second Department of Neurology, Institute of Psychiatry and Neurology, Sobieskiego 9, Warsaw, Poland \\ ${ }^{2}$ Department of Clinical and Experimental Pharmacology, Medical University of Warsaw, Banacha 1b, Warsaw, Poland
}

\begin{abstract}
Introduction: Wilson's disease (WD) is treatable by life-long decoppering therapy with the aim of removing excess copper and preventing re-accumulation. Neurological deterioration sometimes follows the initiation of anti-copper treatment. Prior studies have reported this treatment complication in $10-50 \%$ of WD patients treated with d-penicillamine, and in only 3-7\% of WD patients treated with zinc salts. It has been hypothesized that neurological deterioration after d-penicillamine treatment is caused by rapid copper mobilization from tissues, and elevation of toxic copper in serum. Zinc salts reduce intestinal absorption of copper, and they not influence copper balance as rapidly as d-penicillamine.
\end{abstract}

Case report: We present the case of a patient who experienced neurological worsening after initiation of zinc salts, including marked progression of MRI abnormalities. Copper metabolism tests indicated that anti-copper treatment with ZS was effective.

Conclusion: The presently described case supports the findings of previous studies suggesting the possibility of neurological deterioration after treatment with ZS, rather than only with chelating agents. It is possible that in cases with advanced changes in the brain, WD symptoms may progress for several months, despite initiation of anti-copper treatment.

Implications: There is possibility of neurological deterioration after treatment with zinc salts in Wilson's disease patients, not only after therapy with chelating agents. Compliance in Wilson's disease therapy is crucial to avoid neurological deterioration.

\section{Background}

Wilson's disease (WD) is an inherited autosomal recessive disorder that lead to copper accumulation in the liver, brain, cornea, and other organs [1]. WD is treatable by life-long decoppering therapy with the aim of removing excess copper and preventing re-accumulation. This is accomplished with two main groups of drugs: chelating agents, including d-penicillamine (DPA) and trientine, that promote urinary excretion of copper; and zinc salts, including zinc sulphate (ZS), that interfere with intestinal uptake of copper [1].

Among WD patients with neurological symptoms, neurological deterioration sometimes follows the initiation of anti-copper treatment using a chelating agent, particularly DPA. Prior studies have reported this treatment complication in $10-50 \%$ of WD patients treated with DPA [1-5], and in only 3-7\% of WD patients treated with zinc salts [2,3,5]. However, Weiss et al. [2] reported similar frequencies of neurological worsening in groups treated with chelating agents $(9.1 \%)$ and zinc salts $(7.3 \%)$.

Our recent study showed early neurological worsening in 16 out of 143 analyzed patients (11.1\%), impacting only patients who showed neurological symptoms at diagnosis. The mean time from anti-copper treatment initiation to neurological worsening was about 2 months. Neurological deterioration was completely reversible in 53\% and partially reversible in $13 \%$ of patients. Notably, the treatment type (DPA or zinc sulfate) did not impact deterioration. The main predictors of early deterioration were the severity of baseline neurological symptoms, magnetic resonance imaging (MRI) changes of the thalamus and brain stem, and concomitant dopamine-modulating drug treatment [6].

Herein, we report the case of a patient with neurological deterioration after initial therapy with ZS or natural progression of disease symptoms despite therapy initiation.

\section{Case report}

A 35-year-old female was admitted to our department due to a tremor of her hands lasting for one month. She had been diagnosed with presymptomatic WD twelve years earlier during a family screening after her two sisters were diagnosed with WD. At that time, she started taking ZS but stopped treatment after two months without any particular reason. She came to our center again when neurological symptoms occurred.

Correspondence to: Karolina Dzieżyc, MD PhD, Institute of Psychiatry and Neurology, Second Department of Neurology, Sobieskiego 9, Warsaw, Poland, E-mail: kdziezyc@op.pl

Key words: Wilson's disease, anti-copper treatment, zinc salts, neurological worsening

Received: December 04, 2016; Accepted: December 22, 2016; Published: December 26, 2016 
Upon admission, neurological examination revealed extrapyramidal dysarthria and mild tremor of the left hand (7 points on the Unified Wilson's Disease Score Scale; UWDRS) [7]. Slit lamp examination revealed Kayser-Fleischer rings. Laboratory tests indicated a mildly elevated alanine aminotransferase (ALT) level of $75 \mathrm{U} / 1$ (normal: $<33$ U/l). Copper metabolism testing showed impaired copper metabolism typical for $\mathrm{WD}$, including low serum ceruloplasmin, low total serum copper, increased non-ceruloplasmin-bound copper (NCC), and increased urinary copper excretion (Table 1). Genetic testing confirmed the diagnosis, revealing the H1069Q mutation in one allele and the p.A1135fs mutation in the other allele of the ATP7B gene. T2weighted MRI of the head showed findings typical of WD, including hyperintense lesions in the thalami and midbrain, and discrete lesions in the putamen (Figure 1A and 1B). ZS treatment was initiated with $180 \mathrm{mg}$ of elementary zinc every $24 \mathrm{~h}$.

Over the six months following ZS treatment initiation, the patient showed substantial progression of neurological symptoms. Neurological examination at the six-month follow-up revealed hypomimia; worsening of dysarthria; and new signs, including upper and lower limb rigidity, dystonic posture of right hand, mild right foot dystonia, and dystonic gait (54 points on the UWDRS) [7]. MRI of the head revealed progression of changes in the putamen and midbrain (Figure 1C and 1D). The patient presented no infections or other concomitant diseases, and was undergoing no additional treatment. The patient declared compliance with ZS treatment, which was confirmed by copper metabolism tests (Table 1). At an additional six months after this deterioration, her neurological state remains without improvement.

\section{Discussion}

Here we present the case of a patient who experienced neurological worsening after initiation of ZS, including marked progression of MRI abnormalities. Copper metabolism tests indicated that anti-copper
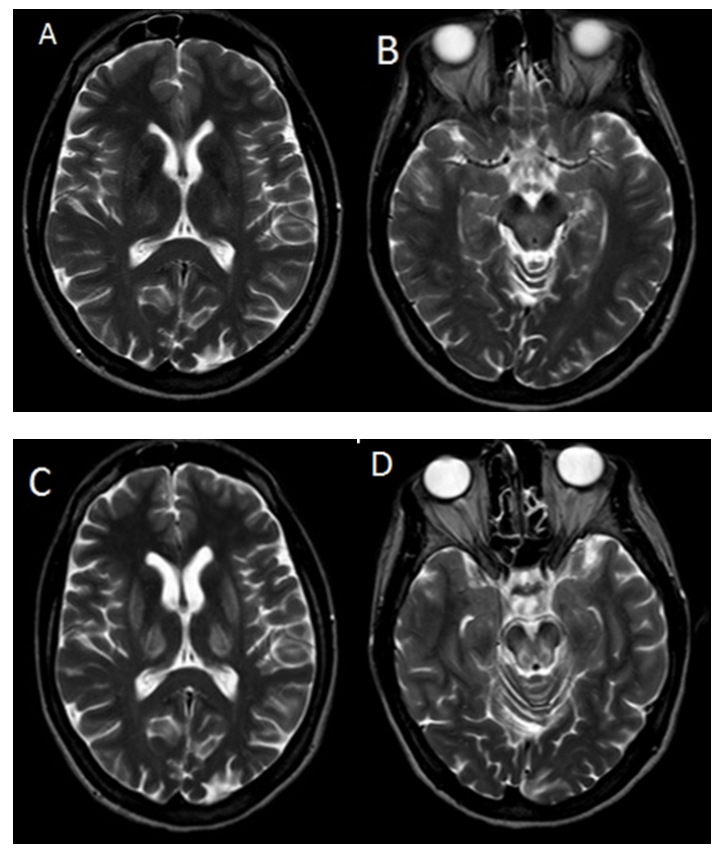

Figure 1. Axial T2-weighted MRI of the brain in July of 2014 showing hyperintense changes in thalami, and discrete lesions in the putamen (A) and in the dorsal part of the midbrain tegmentum (B) Follow-up MRI in February of 2015 showing clinical deterioration (C) with more pronounced hyperintense changes in thalami (D) the putamen, and the midbrain.
Table 1. Results of copper metabolism tests in WD patient with neurological deterioration.

\begin{tabular}{|l|l|l|l|}
\hline & Diagnosis & $\begin{array}{l}\text { Three-month } \\
\text { follow-up }\end{array}$ & $\begin{array}{l}\text { Six-month } \\
\text { follow-up }\end{array}$ \\
\hline $\begin{array}{l}\text { Serum ceruloplasmin }(\mathrm{mg} / \mathrm{dl}) \text {; normal range, } \\
25-45\end{array}$ & 3.4 & 3.3 & 2.7 \\
\hline $\begin{array}{l}\text { Total serum copper }(\mu \mathrm{g} / \mathrm{dl}) \text {; normal range, } \\
70-140\end{array}$ & 27 & 22.5 & 17.4 \\
\hline $\begin{array}{l}\text { Calculated NCC* } \\
(\mu \mathrm{g} / \mathrm{dl})\end{array}$ & 16.8 & 12.6 & 9.3 \\
\hline $\begin{array}{l}\text { Urinary copper excretion }(\mu \mathrm{g} / 24 \mathrm{~h}) \text {; normal } \\
\text { range, } 0-50\end{array}$ & 189 & 139.4 & 133 \\
\hline $\begin{array}{l}\text { Zinc serum copper }(\mu \mathrm{g} / \mathrm{dl}) ; \text { normal range, } \\
50-120^{* *}\end{array}$ & - & 193 & 176 \\
\hline
\end{tabular}

*NCC; non-ceruloplasmin-bound copper, European and American recommendations state that the normal range for free copper is $10-15 \mu \mathrm{g} / \mathrm{dl}$.

**Zinc serum concentration should be above $120 \mu \mathrm{g} / \mathrm{dl}$ in ZS-treated patients.

treatment with ZS was effective, showing normalization of serum NCC, decreased urinary copper excretion, and a therapeutic serum zinc concentration [1].

To date, there have been reports of neurological deterioration after zinc salt treatment $[2,3,5]$, with a few cases described in detail $[8,9]$. The first such reported patient was treated with DPA for 14 years before zinc salts were introduced, and was non-compliant with treatment for about three years. Thus, it was difficult to conclude whether neurological worsening was related to zinc salt treatment [8]. In the second reported case, the patient's neurological condition deteriorated after 8 months of zinc salt therapy, and improved after introduction of a chelating agent [9].

It has been hypothesized that neurological deterioration after DPA treatment is caused by rapid copper mobilization from tissues, and elevation of toxic NCC levels in serum and cerebrospinal fluid [4]. Zinc salts have a different mode of action, involving reduction of intestinal absorption of copper, such that ZS treatment does not influence copper balance as rapidly as DPA $[1,2]$. The mechanisms of neurological deterioration after ZS treatment remain unclear. Since ZS acts more slowly than DPA, the observed neurological deterioration may reflect the continued progression of WD signs, not directly influenced by ZS therapy. At the time of starting anti-copper treatment, our patient already showed advanced changes on brain MRI, affecting the putamen, thalami, and midbrain. Our previous study showed that advanced changes on MRI (especially in the thalami and midbrain) were associated with early neurological deterioration [6].

In the described case, we decided not to change the anticopper treatment, because the copper metabolism tests results were appropriate for the stage in the disease course, and because the patient's neurological status stabilized. This case highlights the necessity of compliance in WD therapy. Our previous study confirmed that anticopper treatment prevents progression to clinically overt WD in compliant presymptomatic patients [10].

\section{Conclusion}

In conclusion, the presently described case supports the findings of previous studies suggesting the possibility of neurological deterioration after treatment with ZS, rather than only with chelating agents. It is possible that in cases with advanced changes in the brain, WD symptoms may progress for several months, despite initiation of anticopper treatment.

\section{Conflicts of interest}

The authors have NO affiliations with or involvement in any 
organization or entity with any financial interest (such as honoraria; educational grants; participation in speakers' bureaus; membership, employment, consultancies, stock ownership, or other equity interest; and expert testimony or patent-licensing arrangements), or nonfinancial interest (such as personal or professional relationships, affiliations, knowledge or beliefs) in the subject matter or materials discussed in this manuscript.

\section{References}

1. European Association for Study of Liver (2012) EASL Clinical Practice Guidelines: Wilson's disease. J Hepatol 56: 671-685. [Crossref]

2. Weiss KH, Gotthardt DN, Klemm D, Merle U, Ferenci-Foerster D, et al. (2011) Zinc monotherapy is not as effective as chelating agents in treatment of Wilson disease. Gastroenterology 140: 1189-1198. [Crossref]

3. CzA,onkowska A, Litwin T, Karliaski M, Dziezyc K, Chabik G, et al. (2014) D-penicillamine versus zinc sulfate as first-line therapy for Wilson's disease. Eur J Neurol 21: 599-606. [Crossref]
4. Brewer GJ (1999) Penicillamine should not be used as initial therapy in Wilson's disease. Mov Disord 14: 551-554. [Crossref]

5. Czlonkowska A, Gajda J, Rodo M (1996) Effects of long-term treatment in Wilson's disease with D-penicillamine and zinc sulphate. J Neurol 243: 269-273. [Crossref]

6. Litwin T, Dzieayc K, Karli A, ski M, Chabik G, Czepiel W, et al. (2015) Early neurological worsening in patients with Wilson's disease. J Neurol Sci 355: 162-167. [Crossref]

7. CzA, onkowska A, Tarnacka B, Maller JC, Leinweber B, Bandmann O, et al. (2007) Unified Wilson's Disease Rating Scale - a proposal for the neurological scoring of Wilson's disease patients. Neurol Neurochir Pol 41: 1-12. [Crossref]

8. Lang CJ, Rabas-Kolominsky P, Engelhardt A, Kobras G, Konig HJ (1993) Fata deterioration of Wilson's disease after institution of oral zinc therapy. Arch Neurol 50: 1007-1008. [Crossref]

9. Walshe JM, Munro NA (1995) Zinc-induced deterioration in Wilson's disease aborted by treatment with penicillamine, dimercaprol, and a novel zero copper diet. Arch Neurol 52: 10-11. [Crossref]

10. Dziezyc K, Karlinski M, Litwin T (2014) Compliant treatment with anti-copper agents prevents clinically overt Wilson's disease in pre-symptomatic patients. Eur J Neurol 21: $332-337$.

Copyright: $\odot 2016$ Dzieżyc K. This is an open-access article distributed under the terms of the Creative Commons Attribution License, which permits unrestricted use, distribution, and reproduction in any medium, provided the original author and source are credited. 